\section{Uso de trombolíticos e alternativas terapêuticas no paciente grave}

Grandes registros mostram que a mortalidade intra-hospitalar por TEP atinge 30\% em pacientes que apresentam eventos agudos associados à instabilidade hemodinâmica ou choque.(1) Estudos usando ecocardiografia têm demonstrado que a presença de DVD é um fator prognóstico importante em pacientes com TEP, mesmo quando normotensos à apresentação, estando presente em 31-56\% dos casos. ${ }^{(2,3)} \mathrm{Um}$ grande registro prospectivo de TEP (International Cooperative Pulmonary Embolism Registry) mostrou que pacientes normotensos tinham chance duas vezes maior de morrer quando apresentavam DVD. ${ }^{(4)} \mathrm{A}$ dilatação do VD detectada por TC (definida como uma razão do diâmetro do VD sobre o diâmetro do VE > 0,9) é vista em 64\% dos pacientes com TEP e também está associada a uma maior chance de morte por TEP em 30 dias (OR $=5,17 ; p=0,005)$, após ajustes para efeito de outros fatores. ${ }^{(5)}$

Esses dados têm suscitado vários estudos com o uso de agentes trombolíticos, desde os anos 70, visando mostrar que uma resolução mais rápida dos êmbolos pulmonares leva à melhora da perfusão pulmonar, detectada por angiografia ou cintilografia pulmonar, e dos parâmetros hemodinâmicos, como DVD. ${ }^{(6)}$ Isso ocorre porque, enquanto a anticoagulação sistêmica com heparina previne apenas a recorrência da TEP, com a remoção parcial do êmbolo, a trombólise leva à redução da sobrecarga e à reversão da dilatação do VD e, em teoria, poderia resultar em uma redução da mortalidade. Entretanto, após um estudo inicial em 1970, apenas 10 outros estudos clínicos controlados foram realizados, com randomização de um total de 717 pacientes, comparando o uso de heparina com o de trombolíticos. ${ }^{(7-16)}$ Esses estudos mostram a evidência conflitante quanto à superioridade de trombolíticos na redução da mortalidade por TEP em pacientes em geral.

Em três meta-análises publicadas nos últimos 5 anos, compilaram-se os dados desses estudos e chegaram a conclusões semelhantes. ${ }^{(17-19)}$ Quando comparados com a heparina, os trombolíticos estão associados a uma redução não significativa na recorrência de TEP ou em morte (6,7\% vs. 9,6\%; OR =0,67; 1C95\%: 0,40-1,12), a um aumento não significativo de sangramento importante $(9,1 \%$ vs. $6,1 \%$; $O R=1,42$; 1C95\%: 0,81-2,46) e a um aumento significativo em sangramentos menores (22,7\% vs. $10,0 \%$; $\mathrm{OR}=2,63$; 1C95\%: 1,53-4,54). Dessa forma, para melhor entendermos a evidência e as indicações para o uso de trombolíticos, é recomendável estratificar os pacientes com TEP de acordo com a presença de instabilidade hemodinâmica (geralmente associada à hipotensão arterial à apresentação) e com a presença de DVD.

Para o primeiro grupo, composto de pacientes com instabilidade hemodinâmica e/ ou choque, apesar da evidência ser tênue, o uso de trombolíticos, na ausência de contraindicações, é recomendado pela maioria dos consensos internacionais, particularmente devido à alta mortalidade associada a essa situação. ${ }^{(20-22)}$ Nesses pacientes, o risco de sangramento grave (levando à hemorragia cerebral ou morte), que normalmente chega a 2-3\%, é claramente superado pelo risco de morte por TEP. ${ }^{(23)} 0$ segundo grupo envolve a situação oposta, na qual pacientes com TEP apresentam-se com pressão arterial, FC e função de VD normais. Nesses casos, os pacientes têm bom prognóstico com o uso de anticoagulação sistêmica apenas e, portanto, não está indicada a trombólise, mesmo que tenham TEP anatomicamente grande. Por fim, há o grupo de pacientes para os quais há bastante controvérsia quanto à indicação de trombolíticos. São aqueles que se apresentam hemodinamicamente estáveis, mas com sinais de DVD. Alguns ensaios clínicos controlados sugerem que esses pacientes podem se beneficiar da resolução mais rápida do êmbolo, com melhora da função do VD. Entretanto, apesar de que a dilatação e a hipocinese de VD estão associadas a um pior prognóstico, a avaliação desses parâmetros é, na maioria das vezes, qualitativa, podendo não ser reprodutível fora do ambiente de ensaios clínicos. Além disto, a presença de DVD pode representar apenas um subgrupo de pacientes previamente mais doentes por outras causas (por ex., insuficiência cardíaca congestiva ou cor pulmonale crônico) e não por uma consequência de TEP.

Finalmente, a determinação de estabilidade hemodinâmica não se baseia em um único parâmetro, como a pressão arterial, mas depende de vários fatores, o que pode levar à inclusão de pacientes com prognósticos muito diversos 
nesse grupo. A heterogeneidade e a relativamente baixa mortalidade desse grupo dificulta a demonstração de benefícios clínicos, pois a amostragem de estudos clínicos randomizados tem que ser muito maior. Em 2002, foi realizado o maior estudo controlado até então, incluindo 256 pacientes com TEP e DVD. ${ }^{16)} 0$ desfecho principal de morte ou escalonamento na terapia ocorreu em 24,6\% dos pacientes randomizados para o uso de heparina e em 11\% daqueles tratados com rtPA $(p=0,006)$. Entretanto, esse desfecho combinado não foi suficientemente objetivo, e não houve diferenças significativas nas incidências de morte ou de recorrência de TEP $(2,2 \%$ vs. $3,4 \%$ para heparina vs. rtPA, e $2,9 \%$ vs. $3,4 \%$ para heparina vs. rtPA, respectivamente), que são as razões mais importantes para o uso de trombolíticos.

De um modo geral, as doses de trombolíticos recomendadas para TEP são as seguintes: estreptoquinase, $250.000 \mathrm{Ul} \mathrm{em} \mathrm{dose} \mathrm{inicial,} \mathrm{seguida} \mathrm{de}$ $100.000 \mathrm{Ul} / \mathrm{h}$ por $24 \mathrm{~h}$; uroquinase, $4.400 \mathrm{Ul} / \mathrm{kg}$ de peso em dose inicial, seguida de $2.200 \mathrm{Ul} / \mathrm{kg}$ por $12 \mathrm{~h}$; ou rtPA i.v., $100 \mathrm{mg} / 2 \mathrm{~h}$ (Quadro 13). ${ }^{(20)} A$ infusão deve ser feita sistemicamente, uma vez que o efeito da lise de coágulos de fibrina é global, e a infusão por cateter pode aumentar o risco de sangramento no local da punção. A duração da infusão pode ser curta, pois tanto a estreptoquinase (1.500.000 UI) quanto rtPA (100 mg) levam à dissolução do êmbolo e à redução da resistência arterial pulmonar quando infundidas em $2 \mathrm{~h}$, sem o aumento do risco de sangramento importante. ${ }^{(24)}$ Heparina pode ser administrada concomitantemente com rtPA, mas não com estreptoquinase ou uroquinase. 0 tempo máximo para o tratamento trombolítico de TEP, diferentemente daquele estabelecido para $1 A M$ e para $A V C$, não é tão limitado, uma vez que o seu uso muitas vezes ocorre como o último recurso no caso de instabilidade hemodinâmica e de eminência de morte, até 2 semanas após o início dos sintomas. Entretanto, sabe-se que quanto mais precoce é a administração do trombolítico, maior a probabilidade de haver reperfusão. No caso de forte suspeita clínica de TEP maciça e de morte eminente, uma dose de $50 \mathrm{mg}$ de rtPA em infusão rápida está indicada durante a ressuscitação cardiorrespiratória. ${ }^{(25)}$

Na decisão do uso de trombolíticos, devem ser respeitadas as contraindicações e balanceada
Quadro 13 - Regimes trombolíticos utilizados em TEP aguda.

\begin{tabular}{|c|c|}
\hline $\begin{array}{c}\text { Agente } \\
\text { trombolítico }\end{array}$ & Dosagem \\
\hline Estreptoquinase & $\begin{array}{l}\text { - } 1.500 .000 \mathrm{Ul} \text { i.v em } 2 \mathrm{~h}^{\mathrm{a}} \\
\text { - } 250.000 \mathrm{Ul} \text { em } 30 \mathrm{~min} \text {, seguido } \\
\text { por } 100.000 \mathrm{Ul} / \mathrm{h} \text { por } 12-24 \mathrm{~h}\end{array}$ \\
\hline $\begin{array}{l}\text { Alteplase } \\
(\mathrm{rtPA})^{\mathrm{b}}\end{array}$ & $\begin{array}{l}\bullet 100 \mathrm{mg} \text { i.v. em } 2 \mathrm{~h}^{\mathrm{a}} \\
\bullet 0,6 \mathrm{mg} / \mathrm{kg} \text { (máximo, } 50 \mathrm{mg} \text { ) i.v. em } \\
15 \mathrm{~min}\end{array}$ \\
\hline
\end{tabular}

aRegime preferencial. 'bara o uso durante parada cardiorrespiratória (ritmo de atividade elétrica sem pulso com forte suspeita de TEP): alteplase, $100 \mathrm{mg}$ i.v. em $15 \mathrm{~min}$.

a relação risco-benefício diante da situação do paciente (Quadro 14). ${ }^{(26,27)}$

Em relação ao uso de trombolíticos na TEP, recomenda-se:

1) Trombolíticos (estreptoquinase ou rtPA) estão indicados para o tratamento de pacientes com TEP maciça e/ou instabilidade hemodinâmica e sem contraindicações importantes para anticoagulantes.(B)

2) Trombolíticos não estão indicados em pacientes com TEP de qualquer tamanho, quando hemodinamicamente estáveis $\mathrm{e}$ sem evidência de DVD.(A)

3) Uma dose de $50 \mathrm{mg}$ de rtPA em bolus pode ser usada emergencialmente em pacientes com forte suspeita clínica de TEP maciça e risco de morte iminente.(D)

4) Na ausência de contraindicações, pacientes com TEP, hemodinamicamente estáveis e com evidência de DVD podem ter um melhor curso clínico intra-hospitalar com o do uso de trombolíticos.(B) Entretanto, trombolíticos não devem ser utilizados em todos os pacientes com essas características, pois benefícios em termos de menor recorrência e de menor mortalidade em relação à anticoagulação ainda não foram comprovados.(B)

Pacientes com TEP grave, quando se cogita o uso de trombolíticos, necessitam obviamente de suporte respiratório e hemodinâmico. (C) A reversão da hipoxemia pode exercer um efeito vasodilatador benéfico sobre a circulação pulmonar. A oxigenoterapia, portanto, tem um papel significativo. Caso seja necessário o uso de ventilação mecânica, deve-se considerar a possibilidade de efeitos hemodinâmicos deletérios pela pressão positiva. 
Quadro 14 - Contraindicações para o uso de trombolíticos.

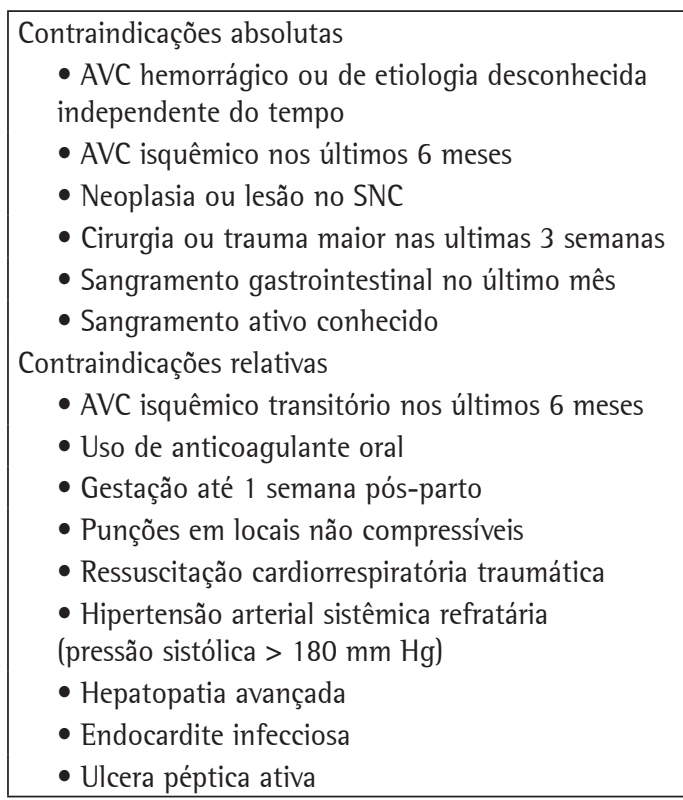

Adaptado de estudos anteriores. ${ }^{(26,27)}$

A insuficiência ventricular direita aguda (ou agudizada) é o mecanismo principal de óbito em pacientes com TEP. Realizar um desafio hídrico agressivo pode piorar a disfunção ventricular. Sendo assim, o uso judicioso de reposição volêmica para otimizar a pré-carga e o débito cardíaco pode ser tentado inicialmente (em geral, 250-500 mL de solução salina). Vários fármacos vasoativos (dobutamina, dopamina, noradrenalina, adrenalina, levosimendana, óxido nítrico, sildenafil e prostaglandinas) já foram testados em modelos experimentais e em pequenas séries de casos. Enfim, o manejo da instabilidade cardiovascular é importante, mas não há uma estratégia comprovadamente eficaz, devendo-se individualizar conforme o contexto do paciente. ${ }^{(28)}$

Aqueles pacientes que não melhoram após uma primeira infusão de trombolítico e permanecem instáveis podem responder a uma segunda infusão de resgate de um outro trombolítico (por ex., se recebeu estreptoquinase, na sequência, seria administrado alteplase e vice-versa). ${ }^{(29)}$

Pacientes graves, com instabilidade hemodinâmica significativa, que têm contraindicações formais a trombolíticos ou não responderam a essa terapia, são candidatos a embolectomia. ${ }^{(29)}$ Esse procedimento pode ser feito através de radiologia intervencionista (por cateter e através de fragmentação e aspiração) ou por cirurgia aberta (com esternotomia e circulação extracorpórea). Entretanto, esses procedimentos são de exceção e necessitam de um centro médico de referência, que não é disponível em muitos locais do Brasil. A embolectomia por cateter visa reduzir agudamente a resistência vascular pulmonar, podendo haver rápida melhora hemodinâmica independentemente do resultado angiográfico. ${ }^{(30)}$ Naqueles pacientes sem contraindicações, podem-se usar trombolíticos locais concomitantemente. No passado, a embolectomia cirúrgica era associada á elevadíssimas taxas de mortalidade (cerca de $80 \%$ ), muito relacionadas à seleção dos pacientes (praticamente moribundos). Atualmente, com o manejo agressivo dos pacientes e a decisão precoce por tratamento cirúrgico com indicação precisa, os resultados têm sido melhores. ${ }^{(31,32)}$

Portanto, pacientes com TEP grave, instabilidade hemodinâmica e contraindicação para ou refratários à terapia trombolítica (um grupo infrequente e altamente selecionado) são candidatos a embolectomia por cateter ou cirúrgica em centros com experiência nesses procedimentos.(C)

\section{Referências}

1. Konstantinides S, Geibel A, Olschewski M, Heinrich F, Grosser K, Rauber K, et al. Association between thrombolytic treatment and the prognosis of hemodynamically stable patients with major pulmonary embolism: results of a multicenter registry. Circulation. 1997;96(3):882-8.

2. Grifoni S, Olivotto 1, Cecchini P, Pieralli F, Camaiti A, Santoro G, et al. Short-term clinical outcome of patients with acute pulmonary embolism, normal blood pressure, and echocardiographic right ventricular dysfunction. Circulation. 2000;101(24):2817-22.

3. Ribeiro A, Lindmarker P, Juhlin-Dannfelt A, Johnsson $\mathrm{H}$, Jorfeldt L. Echocardiography Doppler in pulmonary embolism: right ventricular dysfunction as a predictor of mortality rate. Am Heart J. 1997;134(3):479-87.

4. Goldhaber SZ, Visani L, De Rosa M. Acute pulmonary embolism: clinical outcomes in the International Cooperative Pulmonary Embolism Registry (ICOPER). Lancet. 1999;353(9162):1386-9.

5. Schoepf UJ, Kucher N, Kipfmueller F, Quiroz R, Costello $\mathrm{P}$, Goldhaber SZ. Right ventricular enlargement on chest computed tomography: a predictor of early death in acute pulmonary embolism. Circulation. 2004;110(20):3276-80.

6. The urokinase pulmonary embolism trial. A national cooperative study. Circulation. 1973;47(2 Suppl):111-108.

7. Urokinase pulmonary embolism trial. Phase 1 results: a cooperative study. JAMA. 1970;214(12):2163-72. 
8. Tibbutt DA, Davies JA, Anderson JA, Fletcher EW, Hamill J, Holt JM, et al. Comparison by controlled clinical trial of streptokinase and heparin in treatment of life-threatening pulmonary embolism. Br Med $\mathrm{J}$. 1974;1(5904):343-7.

9. Ly B, Arnesen H, Eie H, Hol R. A controlled clinical trial of streptokinase and heparin in the treatment of major pulmonary embolism. Acta Med Scand. 1978;203(6):465-70.

10. Marini C, Di Ricco G, Rossi G, Rindi M, Palla R, Giuntini C. Fibrinolytic effects of urokinase and heparin in acute pulmonary embolism: a randomized clinical trial. Respiration. 1988;54(3):162-73.

11. Tissue plasminogen activator for the treatment of acute pulmonary embolism. A collaborative study by the PIOPED Investigators. Chest. 1990;97(3):528-33.

12. Levine M, Hirsh J, Weitz J, Cruickshank M, Neemeh J, Turpie AG, et al. A randomized trial of a single bolus dosage regimen of recombinant tissue plasminogen activator in patients with acute pulmonary embolism. Chest. 1990;98(6):1473-9.

13. Dalla-Volta S, Palla A, Santolicandro A, Giuntini C, Pengo V, Visioli 0, et al. PAIMS 2: alteplase combined with heparin versus heparin in the treatment of acute pulmonary embolism. Plasminogen activator Italian multicenter study 2. J Am Coll Cardiol. 1992;20(3):520-6.

14. Goldhaber SZ, Haire WD, Feldstein ML, Miller M, Toltzis $\mathrm{R}$, Smith JL et al. Alteplase versus heparin in acute pulmonary embolism: randomised trial assessing rightventricular function and pulmonary perfusion. Lancet. 1993;341(8844):507-11.

15. Jerjes-Sanchez C, Ramírez-Rivera A, de Lourdes García M, Arriaga-Nava R, Valencia S, Rosado-Buzzo A, et al. Streptokinase and Heparin versus Heparin Alone in Massive Pulmonary Embolism: A Randomized Controlled Trial. J Thromb Thrombolysis. 1995;2(3):227-229.

16. Konstantinides S, Geibel A, Heusel G, Heinrich F, Kasper W; Management Strategies and Prognosis of Pulmonary Embolism-3 Trial Investigators. Heparin plus alteplase compared with heparin alone in patients with submassive pulmonary embolism. N Engl J Med. 2002;347(15):1143-50.

17. Thabut G, Thabut D, Myers RP, Bernard-Chabert B, Marrash-Chahla R, Mal H, et al. Thrombolytic therapy of pulmonary embolism: a meta-analysis. J Am Coll Cardiol. 2002;40(9):1660-7.

18. Agnelli G, Becattini C, Kirschstein T. Thrombolysis vs heparin in the treatment of pulmonary embolism: a clinical outcome-based meta-analysis. Arch Intern Med. 2002;162(22):2537-41.

19. Wan S, Quinlan DJ, Agnelli G, Eikelboom JW. Thrombolysis compared with heparin for the initial treatment of pulmonary embolism: a meta-analysis of the randomized controlled trials. Circulation. 2004;110(6):744-9.

20. Büller HR, Agnelli G, Hull RD, Hyers TM, Prins MH, Raskob GE. Antithrombotic therapy for venous thromboembolic disease: the Seventh ACCP Conference on Antithrombotic and Thrombolytic Therapy. Chest.
2004;126(3 Suppl):401S-428S. Erratum in: Chest. 2005;127(1):416.

21. Guidelines on diagnosis and management of acute pulmonary embolism. Task Force on Pulmonary Embolism, European Society of Cardiology. Eur Heart J. 2000;21(16):1301-36.

22. British Thoracic Society Standards of Care Committee Pulmonary Embolism Guideline Development Group. British Thoracic Society guidelines for the management of suspected acute pulmonary embolism. Thorax. 2003;58(6):470-83.

23. Kanter DS, Mikkola KM, Patel SR, Parker JA, Goldhaber SZ. Thrombolytic therapy for pulmonary embolism. Frequency of intracranial hemorrhage and associated risk factors. Chest. 1997;111(5):1241-5.

24. Meneveau N, Schiele F, Metz D, Valette B, Attali $P$, Vuillemenot $A$, et al. Comparative efficacy of a two-hour regimen of streptokinase versus alteplase in acute massive pulmonary embolism: immediate clinical and hemodynamic outcome and one-year follow-up. J Am Coll Cardiol. 1998;31(5):1057-63.

25. Ruiz-Bailén M, Aguayo-de-Hoyos E, Serrano-Córcoles MC, Díaz-Castellanos MA, Fierro-Rosón JL, RamosCuadra JA, et al. Thrombolysis with recombinant tissue plasminogen activator during cardiopulmonary resuscitation in fulminant pulmonary embolism. A case series. Resuscitation. 2001;51(1):97-101.

26. Torbicki A, Perrier A, Konstantinides S, Agnelli G, Galiè $\mathrm{N}$, Pruszczyk $\mathrm{P}$, et al. Guidelines on the diagnosis and management of acute pulmonary embolism: the Task Force for the Diagnosis and Management of Acute Pulmonary Embolism of the European Society of Cardiology (ESC). Eur Heart J. 2008;29(18):2276-315.

27. Kearon C, Kahn SR, Agnelli G, Goldhaber S, Raskob GE, Comerota AJ, et al. Antithrombotic therapy for venous thromboembolic disease: American College of Chest Physicians Evidence-Based Clinical Practice Guidelines (8th Edition). Chest. 2008;133(6 Suppl):454S-545S.

28. Layish DT, Tapson VF. Pharmacologic hemodynamic support in massive pulmonary embolism. Chest. 1997;111(1):218-24.

29. Meneveau N, Séronde MF, Blonde MC, Legalery P, DidierPetit K, Briand F, et al. Management of unsuccessful thrombolysis in acute massive pulmonary embolism. Chest. 2006;129(4):1043-50.

30. Kucher N, Windecker S, Banz Y, Schmitz-Rode T, Mettler D, Meier B, et al. Percutaneous catheter thrombectomy device for acute pulmonary embolism: in vitro and in vivo testing. Radiology. 2005;236(3):852-8.

31. Yalamanchili K, Fleisher AG, Lehrman SG, Axelrod $\mathrm{HI}$, Lafaro RJ, Sarabu MR, et al. Open pulmonary embolectomy for treatment of major pulmonary embolism. Ann Thorac Surg. 2004;77(3):819-23; discussion 823.

32. Leacche M, Unic D, Goldhaber SZ, Rawn JD, Aranki SF, Couper GS, et al. Modern surgical treatment of massive pulmonary embolism: results in 47 consecutive patients after rapid diagnosis and aggressive surgical approach. J Thorac Cardiovasc Surg. 2005;129(5):1018-23. 